\title{
NO DIVISION IMPLIES CHAOS
}

BY

\author{
TIEN-YIEN LI, MICHAL MISIUREWICZ, GIULIO PIANIGIANI AND
}

JAMES A. YORKE

\begin{abstract}
Let $I$ be a closed interval in $R^{1}$ and $f: I \rightarrow I$ be continuous. Let $x_{0} \in I$ and

$$
x_{i+1}=f\left(x_{i}\right) \text { for } i>0 .
$$

We say there is no division for $\left(x_{1}, x_{2}, \ldots, x_{n}\right)$ if there is no $a \in I$ such that $x_{j}<a$ for all $j$ even and $x_{j}<a$ for all $j$ odd. The main result of this paper proves the simple statement: no division implies chaos.

Also given here are some converse theorems, detailed estimates of the existing periods, and examples which show that, under our conditions, one cannot do any better.
\end{abstract}

1. Introduction. Let $I$ be a closed interval in $R^{1}$ and $f: I \rightarrow I$ be a continuous function. A point $x \in I$ is said to be a point of period $n$ if $f^{n}(x)=x$ and $f^{k}(x) \neq x$ for $1 \leqslant k<n$. For brevity, we will say property $P(k)$ holds if $f$ has a periodic orbit with period $k$. The theorem by A. N. Sharkovski [5] answered the general question of when the existence of a point of period $n$ implies the existence of points of peroid $l$, that is, when $P(n) \Rightarrow P(l)$. The proof of this theorem was considerably shortened in [2].

It is not always immediately apparent whether a periodic orbit of period $n$ exists. In [4], this uncertainty was overcome by use of a more robust hypothesis. We say $\left(x_{0}, x_{1}, \ldots, x_{n}\right)$ is a trajectory if

$$
x_{i+1}=f\left(x_{i}\right)
$$

for $i=0,1, \ldots, n-1$. It was proved there [4] that if there is a trajectory $\left(x_{0}, \ldots, x_{n}\right)$ satisfying either

$$
x_{n} \leqslant x_{0}<x_{1} \text { or } x_{n} \geqslant x_{0}>x_{1}
$$

with $n$ odd, then $P(k)$ holds for some $k$ where $n / k$ is an integer. In [4] we show that $P(k)$ for odd $k>1$ implies "chaos" and we discuss the meaning of chaos.

The main purpose of this paper is to study situations for which $n$ is even in (1.2) and yet there still must be an orbit of odd period. For this, we introduce the notion of 'no division'. For the $n$-tuple $\left(x_{0}, x_{1}, \ldots, x_{n}\right) \subset I$ we say that there is no division

Received by the editors May 8, 1981 and, in revised form, July 21, 1981.

AMS (MOS) subject classifications (1970). Primary 28A65.

Key words and phrases. No division, periodic orbit, chaos, entropies.

'This research was supported by the National Science Foundation under grants MCS 78-02420 (Li) and MCS 78-18221 A01 (Yorke).

This paper was made possible by University of Warwick's invitations to Misiurewicz, Pianigiani and Yorke and by a University of Marlyland invitation to Misiurewicz and Guggenheim fellowship to Yorke. 
if there is no $a \in I$ such that either $x_{j}<a$ for all $j$ even and $x_{j}>a$ for all $j$ odd or $x_{j}>a$ for all $j$ even and $x_{j}<a$ for all $j$ odd. In $\$ 2$, we prove that if $\left(x_{0}, \ldots, x_{n}\right)$ is a trajectory which has no division and satisfies (1.2), then $P(k)$ holds for some odd $k$. In $\S 3$, we obtain better estimates of the existing periods under the above conditions when $n$ is even. In $\S 4$, we give examples to show that under the assumptions of our theorems we cannot obtain stronger results.

2. For future reference, we quote the Sharkovski's theorem in the following:

THEOREM 2.1. Let $f$ be continuous. Then $P(3) \Rightarrow P(5) \Rightarrow P(7) \Rightarrow \cdots \Rightarrow P(2 \cdot 3) \Rightarrow$ $P(2 \cdot 5) \Rightarrow \cdots \Rightarrow P\left(2^{2} \cdot 3\right) \Rightarrow P\left(2^{2} \cdot 5\right) \Rightarrow \cdots \Rightarrow P\left(2^{3}\right) \Rightarrow P\left(2^{2}\right) \Rightarrow P(2) \Rightarrow P(1)$.

For what follows, we let $x_{k}=f^{k}\left(x_{0}\right)$ for all $k \geqslant 0$.

PROPOSITION 2.2. Let $x_{n} \leqslant x_{0}<x_{1}$, or $x_{n} \geqslant x_{0}>x_{1}$, and let there be no division for the trajectory $\left(x_{0}, x_{1}, \ldots, x_{n}\right)$. Then there exists a periodic point of period

(1) $n$ if $n$ is odd,

(2) $n-1$ if $n$ is even.

Proof. For simplicity, we only consider $x_{n} \leqslant x_{0}<x_{1}$. The other case follows by reversing signs in the argument. Let $X=\left\{x_{0}, \ldots, x_{n-1}\right\}$. Since $f\left(x_{0}\right)>x_{0}$ and $f(\max X)<\max X$, the set $S=\left\{x \in X: x>x_{0}, f(x)<x\right\}$ is nonempty. Let $x_{i}=$ $\min S$ and $x_{j}=\max \left\{x \in X: x<x_{i}\right\}$. Then, for $J=\left[x_{j}, x_{i}\right]$ we have int $J \cap X=\varnothing$ and $f(J) \supset J$. Since $x_{n}=f^{n-j}\left(x_{j}\right)$ is in $f^{n-j}(J)$ and $x_{j}$ is in $J \subset f^{n-j}(J)$, and $x_{n} \leqslant x_{0} \leqslant x_{j}$, we have $x_{0} \in f^{n-j(}(J)$. Therefore, all elements of $X$, except perhaps $x_{j-1}\left(\right.$ or $x_{n-1}$ if $j=0$ ) are in $\cup_{i=0}^{n-2} f^{i}(J)$. Since $f(J) \supset J, f^{n-2}(J) \supset f^{n-1}(J) \supset$ $\cdots \supset f(J) \supset J$, hence $\cup_{i=0}^{n-2} f^{n}(J)=f^{n-2}(J)$. We may replace $x_{j}$ with $x_{i}$ in the above argument, and conclude that every point of $X$ except perhaps $x_{i-1}$ is in $f^{n-2}(J)$. Since $i \neq j$, we have $f^{n-2}(J) \supset X$.

Since there is no division, there exists an interval $K=[c, d]$ such that $c, d \in X$ and $(c, d) \cap X=\varnothing$ and $c$ and $d$ lie on the same side of $J$ but $f(c)$ and $f(d)$ lie on the opposite sides of $J$. Therefore $f(K) \supset J$ and $K$ and $J$ have at most one point in common. We thus have the following:

(1) $f(J) \supset J$,

(2) $f(K) \supset J$,

(3) $f^{n-2}(J) \supset K$.

To find periodic points we use the technique from [1] and [2].

From (2) and (3), there is a point $x \in J$, with $f^{n-1}(x)=x$ and $f^{n-2}(x) \in K$. From (1), (2) and (3), there is a point $y \in J$ with $f^{n}(y)=y$ and $f(y) \in J$ and $f^{n-1}(y) \in K$. Neither $x$ nor $y$ are fixed points of $f$, because they would then be common endpoints of $J$ and $K$. Such a common endpoint (if it exists) is an element of $X$ and lies to the right of (or is equal to) $x_{0}$. Some iterate of it is equal to $x_{n}$, and therefore, it cannot be a fixed point.

If $n$ is odd (resp. even) then $y$ (resp. $x$ ) is a peroidic point whose period is a factor of $n$ (resp. $n-1$ ). Hence, by Theorem 2.1, there exists a periodic point of period $n$ (resp. $n-1)$. 
REMARK. If $n$ is odd and $x_{n} \leqslant x_{0}<x_{1}$ or $x_{n} \geqslant x_{0}>x_{1}$ then automatically there is no division for $\left(x_{0}, x_{1}, \ldots, x_{n}\right)$.

As a corollary to Proposition 2.2, we have

THEOREM 2.3. Let $x_{n} \leqslant x_{0}<x_{1}$ or $x_{n} \geqslant x_{0}>x_{1}$ and assume there is no division for $\left(x_{0}, x_{1}, \ldots, x_{n}\right)$. Then there exists a periodic point of odd $(\neq 1)$ period.

The above results show it is sometimes possible to conclude there is a periodic orbit by knowing only the trajectory through one point. We can ask how much improvement is possible in our results. Suppose we have a bounded trajectory $Y=\left(y_{0}, y_{1}, \ldots\right)$. We assume for the remainder of this section that there is at least one continuous $F$ which has $Y$ as a trajectory. Is possible that $Y$ implies the existence of an odd period orbit and yet our results are insufficient for us to be able to conclude this existence?

We say $Y$ implies the existence of an odd period orbit if every continuous $f$ that has $Y$ as a trajectory also has an odd period orbit with period greater than one.

We now prove that the above results can be applied to every (infinite) trajectory $Y$ that implies the existence of an odd period orbit.

THEOREM 2.4. Let $\left(y_{0}, y_{1}, \ldots\right)$ be a sequence which implies the existence of an odd period orbit. Then there exist integers $u$, v such that if $x_{0}=y_{u}, x_{1}=y_{u+1}, \ldots, x_{v}=$ $y_{u+v}$, then there is no division for $\left(x_{0}, \ldots, x_{v}\right)$ and $(1.2)$ is satisfied for $n=v$.

Proof. Denote $B=\left\{y_{0}, y_{1}, \ldots\right\}, J=[\inf B, \sup B]$. It is easy to see that we may assume that $f$ is monotone on components of $J \backslash B$ and constant to the left and to the right of $J$. By assumption, there is a periodic point of $F$ with odd period $n(\neq 1)$. Denote the orbit of this point by $X$. We shall consider the following two cases:

Case 1: $X \subset \bar{B}$.

Choose $x \in X\{\min X, \max X\}$. Without loss of generality we assume $f(x)>x$. We have $f^{k}(x)=f^{n+k}(x)=\min X<x$ for some $k>1$. One of the integers $k$ of $k+n$ is odd. Call this integer $v$. Then, $f^{v}(x)<x<f(x)$. Since $x \in \bar{B}$, we can choose $x_{0} \in B$ with $f^{v}\left(x_{0}\right)<x_{0}<f\left(x_{0}\right)$. By the remark, there is no division for $\left(x_{0}, f^{1}\left(x_{0}\right), f^{2}\left(x_{0}\right), \ldots, f^{v}\left(x_{0}\right)\right)$. This ends the proof in this case.

Case 2: $X \not \subset \bar{B}$.

Since $f(\bar{B}) \subset \bar{B}, X$ is disjoint from $\bar{B}$. Also $f(I) \subset J$, so $X \subset J \backslash \bar{B}$. For any $x \in J \backslash \bar{B}$ let $J_{x}$ denote the component of $J \backslash \bar{B}$ containing $x$. For $c \in X$, we say $c$ is an increasing point if $f(c)>c$. Otherwise, we call $c$ a decreasing point. The orbit of $X$ forms a cycle of $n$ points. Since $n$ is odd, we cannot have alternating increasing and decreasing points. Therefore, there exists $c \in X$ with either $c<f(c)<f^{2}(c)$ or $c>f(c)>f^{2}(c)$. Without loss of generality, we may assume that $c<f(c)<f^{2}(c)$. Since $f$ is monotone on $J_{c}$, the image of one of the endpoints of $J_{c}$ is to the left of $f(c)$. Denote this endpoint by $a$. Therefore $f(a)<f(c)$. Similarly, we can find an endpoint $b$ of $J_{f(c)}$ such that $f(b)>f^{2}(c)$. Clearly, $b \leqslant \sup J_{f(c)} \leqslant \sup J_{f^{2}(c)} \leqslant f(b)$. If $b=f(b)$, then $J_{f(c)}=J_{f^{2}(c)}$ and $b=\sup J_{f(c)}$. It follows that $f([f(c), b])=$ $\left[f^{2}(c), b\right] \subset[f(c), b]$, and $\lim _{n \rightarrow \infty} f^{n}(c)=b$. This contradicts the periodicity of $c$. 
Therefore,

$$
b<f(b)
$$

Suppose that $f(a) \leqslant a \leqslant b$. Since $a \geqslant f(a)$ and $b<f(b)$, we have $a \neq b$. Hence, $f(a) \leqslant a<b<f(b)$.

If $f(a)<a$, then since $a \in \bar{B}$, we can find $x$ and $x^{\prime} \in B$ with $f(x)<x<$ $(a+b) / 2$ and $(a+b) / 2<x^{\prime}<f\left(x^{\prime}\right)$. We have $x=y_{k}, x^{\prime}=y_{m}$ for some $k, m$. Therefore $y_{k+1}<y_{k}<y_{m}<y_{m+1}$. Since the first pair of subscripts contain one odd and one even and the same is true of the final pair, there is no division. The conclusion of the theorem is proved.

We now consider the sole remaining situation: $f(a)=a$.

If $a$ is an isolated point of $\bar{B}$, then $a \in B$ and consequently $a=y_{q}$ for all $q$ sufficiently large. Hence, $y_{q}<y_{m}<y_{m+1}$ for some $m<q$ with $q-m$ being odd. The conclusion of the theorem thus follows. If $a$ is not an isolated point of $\bar{B}$, then there exists $y_{q}$ with arbitrarily large $q$, arbitrarily close to $a$. We may require $q>m$ and $y_{q}$, $f\left(y_{q}\right)<b$. But then one of the integers $q-m$ and $q+1-m$ is odd and again we have the conclusion of the theorem.

The remaining situation is either

$$
f(a)>a \text { or } a>b .
$$

Suppose that $J_{c}=J_{f(c)}$. Since $c<f(c)$ and $f(c)<f^{2}(c), f$ is increasing in $J_{c}$. Therefore, $f(a) \leqslant a=\inf J_{c}<\sup J_{c}=b \leqslant f(b)$ which contradicts (2.2). Hence $J_{c} \neq$ $J_{f(c)}$, and $a, f(a) \leqslant \inf J_{f(c)} \leqslant b$. In view of (2.1) and (2.2), we have

$$
a<f(a) \leqslant b<f(b) \text {. }
$$

Since $a, b \in \bar{B}$, we can choose $x, x^{\prime} \in B$ such that $x<f(x) \leqslant x^{\prime}<f\left(x^{\prime}\right)$. We have $x=y_{k}, x^{\prime}=y_{m}$ for some $k, m$. If we can choose $x, x^{\prime}$ in such a way that $k>m$, then we are done since $y_{k}<y_{k+1} \leqslant y_{m}<y_{m+1}$ and one of the integers $k-m, k+1-m$ is odd. There are only two cases in which we cannot choose such $x$ and $x^{\prime}$ : either $x$ and $x^{\prime}$ cannot be chosen independently, i.e., $f(a)=b$, or there is no $y_{i}$ with large $i$ in some neighborhood of $a$.

Suppose $a \notin B$. Then the second possibility cannot occur, and consequently we are left with $f(a)=b$. We may take three elements of $B$ arbitrarily close to $a: y_{m-1}$, $y_{k}, y_{p}$ such that $m-1<k<p$. Then we have $y_{p}<y_{m}<y_{m+1}$ and $y_{k}<y_{k+1}<y_{k+2}$, and for $y_{m}=x_{0}, \ldots, y_{p}=x_{v}$ there is no division.

If $a \in B$, then we take $x=y_{k}=a$. If $f^{2}(a) \leqslant f(a)$, then $a<b$. We may find $x^{\prime}=y_{m}$ and $y_{k+2} \leqslant y_{k+1}<y_{m}<y_{m+1}$, which has no division.

If $f^{2}(a)>f(a)$, then we have $a=y_{k}<y_{k+1}<y_{k+2}$. Let $K=\left[\inf _{i \geqslant k+1} y_{i}\right.$, $\sup _{i \geqslant k+1} y_{i}$ ]. Since $y_{k+1}=f(a)<f(c)<f^{2}(c)<f\left(x^{\prime}\right)=y_{m+1}$, and $m+1>k+1$, we have $X \cap K$ is nonempty. Hence $c \in f^{p}(K)$ for some $p$. Since $f$ is monotone on components of $J \backslash \bar{B}$, there exists $z \in B \cap K$ such that $f^{p}(z) \leqslant \inf J_{c} \leqslant a$. We have $z=y_{s}$ for some $s$. If $s \geqslant k+1$, then $f^{p}(z)=y_{s+p}$ and $s+p>k+1$. It follows that $y_{s+p} \leqslant y_{k}<y_{k+1}<y_{k+2}$ which has no division because of the last three terms. If $s<k+1$ then $y_{t}<y_{s}<y_{r}$ for some $t, r \geqslant k+1>s$. The result of the theorem is proved no matter where $y_{s+1}$ is, unless either $t=k+1$ and $y_{s}<y_{s+1}$, or $r=k+1$ 
and $y_{s+1}<y_{s}$. In the first case, we get $y_{k}<y_{k+1}<y_{s}<y_{s+1}$ and $s<k$, and in the second case $y_{s+1}<y_{s}<y_{k}<y_{k+1}$ and $s<k$, which are cases where there is no division.

3. Let $f: I \rightarrow I$ be continuous and $x_{i}=f^{i}\left(x_{0}\right)$ for all $i \geqslant 1$. In this section, we obtain better estimates of the periods for the case where $x_{n} \leqslant x_{0}<x_{1}$ or $x_{n} \geqslant x_{0}>x_{1}$ with even $n$ and there is no division for $\left(x_{0}, x_{1}, \ldots, x_{n}\right)$. We first prove the following lemma:

LEMMA 3.1. Let $x_{n+1} \leqslant x_{n}<x_{0} \leqslant x_{1}$ or $x_{n+1} \geqslant x_{n}>x_{0} \geqslant x_{1}$ for some $n>1$. Then $f$ has periodic points of all periods.

Proof. Notice that $x_{0} \neq x_{1}$ since otherwise, $x_{n}=x_{0}$. As usual we shall only consider the case $x_{n+1} \leqslant x_{n}<x_{0}<x_{1}$. Without loss of generality, we may assume that $n$ is the smallest positive integer for which $x_{n+1} \leqslant x_{n}<x_{0}$. Let

$$
m=\min \left\{r \geqslant 0, x_{r+1} \leqslant x_{n}\right\} .
$$

Clearly, $m<n$. If $x_{m}<x_{0}$, then $x_{m+1} \leqslant x_{n}<x_{m}<x_{0}$, which contradicts the definition of $n$. If $x_{m}=x_{0}$ then $x_{m+1}=x_{1}$, but $x_{m+1} \leqslant x_{n}<x_{1}$, a contradiction. Consequently, $x_{m}>x_{0}$. Let $k=\min \left\{r \geqslant 0: x_{r+1} \geqslant x_{m}\right\}$. Then $k<m, x_{k}<x_{m}$ and $x_{k+1} \geqslant x_{m}$. Since $k<m$, we have $x_{k}>x_{n}$. Let $K=\left[x_{n}, x_{k}\right]$ and $M=\left[x_{k}, x_{m}\right]$. Then, $f(K) \supset\left[x_{n+1}, x_{k+1}\right] \supset K \cup M$ and $f(M) \supset\left[x_{m+1}, x_{k+1}\right] \supset K \cup M$. Since $K \cap M=\left\{x_{k}\right\}$ and $x_{k}$ is not a fixed point of $f, f$ has periodic points of all periods.

COROLlaRY 3.2. If $f$ does not have periodic points of all periods, then there exists a fixed point $z$ of $f$ such that if $x_{i}<z$ then $x_{i+1}>x_{i}$, and if $x_{i}>z$ then $x_{i+1}<x_{i}$.

Proof. Suppose that $f$ does not have periodic points of all periods. Then from Lemma 3.1, it follows that if $x_{m+1} \leqslant x_{m}$ and $x_{k+1} \geqslant x_{k}$ then $x_{m} \leqslant x_{k}$. Let $a=$ $\sup \left\{x_{i}: x_{i+1} \geqslant x_{i}\right\}, b=\inf \left\{x_{i}: x_{i+1} \leqslant x_{i}\right\}$. Then $a \leqslant b$. If $f(a)=a$, we may take $z=a$; if $f(b)=b$, we may take $z=b$. If not, then $f(a) \geqslant b$ and $f(b) \leqslant a$ and there is a fixed point $z$ between $a$ and $b$. Clearly, such $z$ has the required properties.

LeMmA 3.3. Let $X=\left(x_{0}, x_{1}, \ldots, x_{n-1}, x_{n}\right)$ have no division and assume $n$ is even and $x_{n}=x_{0}$. Then, $f$ has a periodic orbit of period $n / 2$ if $n / 2$ is odd, period $(n / 2)+1$ if $n / 2$ is even.

Proof. Suppose the conclusion of the lemma is false. Then, by Corollary 3.2 , there is a fixed point $z$ of $f$, such that for any $x \in X$, if $x<z$ then $f(x)>x$ and if $x>z$ then $f(x)<x$. We consider the two cases separately. Since $x_{n}=x_{0}$, we have $x_{i+n}=x_{i}$ for all $i$.

Case 1: $n / 2$ is odd. Suppose that for some $k, x_{k}$ and $x_{k_{*}}$ where $k_{*}=k+n / 2$ are on the same side of $z$. If $x_{k}$ is closer to $z$, then we have $x_{k_{*}}<x_{k}<x_{k+1}$ or $x_{k_{*}}>x_{k}>x_{k+1}$ and we get a periodic point of period $n / 2$, a contradiction. If $x_{k_{*}}$ is closer to $z$, then we can use the same type of argument because $x_{k}=x_{n+k}$ and also get a contradiction. Hence, for all $k, x_{k}$ and $x_{k_{*}}$ are on opposite sides of $z$. We may assume, without loss of generality, that there are no elements of $X$ in $\left(x_{0}, z\right]$. Since 
there is no division for $X$, there exists $d$ odd, $0<d<n$, such that $x_{d}<z$. Hence, $x<x_{0}<z<x_{n / 2}$. We know that $z<x_{d_{*}}$ where $d_{*}=d+n / 2$. If $x_{d_{*}}<x_{n / 2}$ then either $d \leqslant n / 2$ and then $x_{d}<x_{0}<x_{1}$ gives a contradiction, or $d>n / 2$ and then (writing $t=d-n / 2) x_{t+1}<x_{t}<x_{n / 2}$ gives a contradiction. It follows that $x_{d}<x_{0}$ $<z<x_{n / 2}<x_{d_{*}}$. Since $x_{0}<z$, we have $x_{1}>x_{0}$ and consequently $x_{1}>z$. Hence, $x_{n / 2+1}<z$, and $x_{n / 2+1}<x_{0}$. Since $x_{d}<x_{0}<x_{1}$, we must have $d>n / 2$. If $x_{1}<x_{t}$, then $x_{2}<x_{1}<x_{t}$ we get a periodic point of odd period $d-n / 2-1 \leqslant n / 2$, a contradiction $\left(t \neq 1\right.$ since $\left.x_{1}<x_{t}\right)$. Therefore $x_{t}<x_{1}$ (there is no equality since $d-n / 2$ is even). Hence, $x_{1+n / 2}<x_{0}<x_{n / 2}<x_{t}<x_{1}$. Since $n / 2$ is odd and $t$ is even, there is no division for $\left\{x_{0}, x_{1}, \ldots, x_{n / 2+1}\right\}$. By Proposition 2.1, there exists a periodic point of period $n / 2$.

Case 2: $n / 2$ is even. By Theorem 2.1, if there is no periodic point of period $n / 2+1$ then there is no periodic point of period $n / 2-1$. By an argument similar to the one used in Case 1, we establish that every $k, x_{k}$ and $x_{k_{*}-1}$ are on the opposite sides of $z$. Also, $x_{k}$ and $x_{k_{*}+1}$ are on the opposite sides of $z$. Hence, for every $m, x_{m}$ and $x_{m+2}$ are on the same side of $z$. Consequently, either all elements of $x$ are on the same side of $z$, or there is a division for $X$. In both cases we obtain a contradiction.

Proposition 3.4. Let $x_{n} \leqslant x_{0}<x_{1}$ or $x_{n} \geqslant x_{0}>x_{1}$ with $n$ even, and assume there is no division for $\left(x_{0}, x_{1}, \ldots, x_{n}\right)$. Then there exists a periodic point of period $n / 2$ if $n / 2$ is odd, and $n / 2+1$ if $n / 2$ is even.

Proof. Suppose the conclusion of the proposition is false. Write $X=\left\{x_{0}, \ldots, x_{n}\right\}$. By Corollary 3.2, there is a fixed point $z$ of $f$ such that for $x \in X$, if $x<z$ then $f(x)>x$ and if $x>z$ then $f(x)<x$. It is obvious that $z$ is not an element of $X$. We will first find a periodic orbit of period $n$ with no division and then apply Lemma 3.3. For simplicity, we only examine the case $x_{n} \leqslant x_{0}<x_{1}$.

Let $J_{k}$ denote the interval: $\left[x_{k}, z\right]$ if $x_{k}<z$ and $\left[z, x_{k}\right]$ if $x_{k}>z, k=0,1, \ldots, n$. Since $x_{1}>x_{0}$, we have $x_{0}<z$. Hence, $J_{n} \supset J_{0}$. It follows that $f\left(J_{k}\right) \supset J_{k+1}$ for $k=0,1, \ldots, n-2$ and $f\left(J_{n-1}\right) \supset J_{0}$. Since there is no division for $X$, there exists $q \leqslant n-1$ such that $x_{q}, x_{q+1}$ are on the same side of $z$. Look subsequently at the points $x_{q+2}, x_{q+3}, \ldots, x_{n-1}, x_{0}, x_{1}, \ldots, x_{q-1}$. Some of them have to lie on the opposite side of $z$ from $x_{q}$. Let $x_{p}$ be the first such point. Then $x_{p-2}, x_{p-1}$ lie on one side of $z$ and $x_{p}$ is on the opposite side (the indices are interpreted $\bmod n$ ). Suppose $x_{p-2}=x_{p-1}$. If $p-2=n-2$ and $x_{p-1}=x_{p-2}$, then $x_{p-1}$ and $x_{p}$ are on the same side of $z$. If $p-2=n-1$ and $x_{p-2}=x_{p-1}$ (note: $x_{p-1}=x_{0}$ ), then $x_{n}=x_{1}$. If $p-2<n-2$ then $x_{p} \neq x_{p-1}$. In all cases, we get a contradiction. Consequently, $x_{p-2} \neq x_{p-1}$. Let $P$ denote the interval $\left[x_{p-2}, x_{p-1}\right]$ if $x_{p-2}<x_{p-1}$ and $\left[x_{p-1}, x_{p-2}\right]$ if $x_{p-1}>x_{p-2}$.

We have $P \subset J_{p-2}$ and therefore $f\left(J_{p-3}\right) \supset P$. If $p-2<n-2$ then $f(P) \supset$ $\left[x_{p-1}, x_{p}\right]$ (or $\left[x_{p}, x_{p-1}\right]$ ), and since $x_{p-1}$ and $x_{p}$ are on the opposite sides of $z$, $f(P) \supset J_{p-1}$. If $p-2=n-2$ then $f(P) \supset\left[x_{n}, x_{n-1}\right] \supset\left[z, x_{n-1}\right]=J_{p-1}$. If $p-2$ $=n-1$ then $f(P) \supset\left[x_{n}, x_{1}\right] \supset J_{0}=J_{p-1}$. In all cases, we thus have $f(P) \supset J_{p-1}$. It follows that ..., $f\left(J_{p-4}\right) \supset J_{p-3}, f\left(J_{p-3}\right) \supset P, f(P) \supset J_{p-1}, f\left(J_{p-1}\right) \supset J_{p}, \ldots$ and the 
cycle closes. The length of this cycle is $n$. There is a periodic point $y \in P$ such that $f(y) \in J_{p-1}, f^{2}(y) \in J_{p}, \ldots, f^{n-2}(y) \in J_{p-4}, f^{n-1}(y) \in J_{p-3}$, and $f^{n}(y)=y$. Since the intervals $P, J_{p-1}, J_{p}$ have pairwise disjoint interiors and $J_{p-1}$ lies between $P$ and $J_{p}$, we have either $y<f(y)<f^{2}(y)$ or $y>f(y)>f^{2}(y)$. Hence, for the orbit of $y$ there is no division. This orbit has a period $k \geqslant 3$ and $k$ divides $n$. If $k$ is odd then we have $k \leqslant n / 2$ and we use Theorem 2.1. If $k$ is even, then we use Lemma 3.3 and then (if necessary) Theorem 2.1 .

We now investigate what happens if the lack of division occurs "too late" (i.e., after $n$ with $x_{n} \leqslant x_{0}<x_{1}$ ).

PROPOSITION 3.5. Let either $x_{n} \leqslant x_{0}<x_{1}$ and $x_{d} \leqslant x_{p}$ or $x_{n} \geqslant x_{0}>x_{1}$ and $x_{d} \geqslant x_{p}$, for some $d, p, n$ where $d \leqslant n$ and $d$ is odd and $p$ is even. Then there exists a periodic orbit of some odd $(\neq 1)$ period $k \leqslant \max \{n, p-d\}$.

Proof. As usual we only prove the case for $x_{n} \leqslant x_{0}<x_{1}$ and $x_{d} \leqslant x_{p}$. If $n$ is odd we get, by Proposition 2.2 and Remark after it, a periodic point of period $n$. So, we only consider the case where $n$ is even.

Case 1: $x_{d} \leqslant x_{0}$. Then, $x_{d} \leqslant x_{0}<x_{1}$ and we get a periodic point of period $d \leqslant n$ with odd $d$.

Case 2: $x_{d}>x_{0}$. If $x_{d+1}=x_{d}$, then $x_{n}=x_{d}>x_{0}$, a contradiction. If $x_{d+1}>x_{d}$ then $x_{n}<x_{d}<x_{d+1}$ and there is a periodic point of period $n-d<d$ with $n-d$ odd. If $x_{d+1}<x_{d}$ then $x_{d+1}<x_{d} \leqslant x_{p}$. If $p>n$, we get a point of period $p-d$. If $p \leqslant n$, there is no division for $\left(x_{0}, \ldots, x_{n}\right)$. By Proposition 2.2 , there is a periodic point of period $n-1$.

Dropping the hypothesis that $d \leqslant n$, we lose the existence of an odd period.

PROPOSITION 3.6. Let either $x_{n} \leqslant x_{0}<x_{1}$ and $x_{d} \leqslant x_{p}$ or $x_{n} \geqslant x_{0}>x_{1}$ and $x_{d} \geqslant x_{p}$ for some $d, p, n$ with $d$ odd and $p$ even. Then, there exists a periodic point of period 6 .

Proof. If there exists a periodic point of an odd $(\neq 1)$ period then we can use Theorem 2.1. Suppose that there is no periodic point of any odd period bigger than 1 (and, in particular, $n$ must be even). We only consider the case where $x_{n} \leqslant x_{0}<x_{1}$ and $x_{d} \leqslant x_{p}$. We shall use the induction argument on $n$.

Case 1: $n=2$. Since there are no periodic points of odd period, there exists, by Corollary 3.2, a fixed point $z$ of $f$ such that if $x_{i}<z$ then $x_{i+1}>x_{i}$ and if $x_{i}>z$ then $x_{i+1}<x_{i}$. Hence, $x_{2} \leqslant x_{0}<z<x_{1}$. Since $x_{d} \leqslant x_{p}$, we have either $x_{d} \leqslant z$ or $x_{p} \geqslant z$. Hence $z$ does not split the even indices from the odd indices. That is, there exists $k$ such that either $x_{k} \leqslant x_{k+1} \leqslant z$ or $x_{k} \geqslant x_{k+1} \geqslant z$. Take the smallest such $k$. Now we shall use induction on $k$.

Case 1A: $k=2$. In this case we have $x_{2}<x_{0}<z<x_{1}$ and $x_{3} \leqslant z$. Consequently, $f\left(\left[x_{2}, x_{0}\right]\right) \supset\left[z, x_{1}\right]$ and $f\left(\left[x_{0}, z\right]\right) \supset\left[z, x_{1}\right]$ and $f\left(\left[z, x_{1}\right]\right) \supset\left[x_{2}, x_{0}\right] \cup\left[x_{0}, z\right]$. Therefore, $f^{2}\left(\left[x_{2}, x_{0}\right]\right) \supset\left[x_{2}, x_{0}\right] \cup\left[x_{0}, z\right]$ and $f^{2}\left(\left[x_{0}, z\right]\right) \supset\left[x_{2}, x_{0}\right] \cup\left[x_{0}, z\right]$. This gives us the existence of periodic points of all periods for $f^{2}$ and hence the existence of periodic points of all even periods for $f$.

Case 1B: $k \geqslant 3$. We claim we only need to consider

$$
x_{2} \leqslant x_{j} \leqslant x_{1} \text { for all } j=0,1, \ldots, k+1 .
$$


Suppose (3.1) is not true. Then, there is $j \in[3, k+1]$ such that $x_{j}=$ $\min \left\{x_{0}, \ldots, x_{k+1}\right\}$ or $x_{j}=\max \left\{x_{0}, \ldots, x_{k+1}\right\}$. For the first case, we have $x_{j} \leqslant x_{j-2}$ $<x_{j-1}$, and, for the second, $x_{j} \geqslant x_{j-2}>x_{j-1}$. We then use the induction hypothesis for $y_{0}=x_{j-2}, y_{1}=x_{j-1}, \ldots, y_{k-j+2}=x_{k}$, with $k-j+2<k$.

Let $x_{r}=\max \left\{x_{i}: 0 \leqslant i \leqslant k, x_{i}<x_{0}\right\}, x_{s}=\min \left\{x_{i}: 0 \leqslant i \leqslant k, x_{i}>x_{0}\right\}$. Let $t=r$ if $x_{r+1} \geqslant x_{s+1}$ and $t=s$ if $x_{s+1}>x_{r+1}$. Suppose first that $t=k$. If $t=s$ and $x_{s}<z$, then $x_{s+1} \leqslant z<x_{r+1}$ and $t=r$, a contradiction. If $t=s$ and $x_{s}>z$ then $x_{0}<z<x_{r+1}<x_{s+1}<x_{s}$. This contradicts the definition of $s$. If $t=r$, then $x_{r+1} \leqslant z<x_{s+1}$ and $t=s$. This is again a contradiction. Hence $t<k$. If $x_{t+1} \leqslant z$ then $x_{t}>z$ and consequently $t=s$. But then, by the definition of $r$ and $s, x_{t+1} \leqslant x_{r}$ and therefore $x_{r+1}>x_{t+1}$, a contradiction. Hence, $x_{t+1}>z$. Suppose $t=k-1$. Then $x_{t+2} \geqslant z$, and $f\left(\left[x_{r}, x_{0}\right]\right) \supset\left[x_{t+1}, x_{1}\right], f\left(\left[x_{0}, z\right]\right) \supset\left[x_{t+1}, x_{1}\right]$ and $f\left(\left[x_{t+1}, x_{1}\right]\right)$ $\supset\left[x_{r}, x_{0}\right] \cup\left[x_{0}, z\right]$. Hence, $f^{2}\left(\left[x_{r}, x_{0}\right]\right) \supset\left[x_{r}, x_{0}\right] \cup\left[x_{0}, z\right]$ and $f^{2}\left(\left[x_{0}, z\right]\right) \supset$ $\left[x_{r}, x_{0}\right] \cup\left[x_{0}, z\right]$. This gives the existence of period points of all periods for $f^{2}$ and hence, periodic points of all even periods for $f$. Suppose $t<k-1$. Then, by definition of $r$ and $s, x_{t+2} \notin\left(x_{r}, x_{s}\right)$. If $x_{t+2} \leqslant x_{r}$, then $x_{t+2} \leqslant x_{t}<x_{t+1}$ and we may use the induction hypothesis for $y_{0}=x_{t}, \ldots, x_{k-t}=x_{k}$ with $k-t<k$. If $x_{t+2} \geqslant x_{s}$ then $f\left(\left[x_{r}, x_{0}\right]\right) \supset\left[x_{t+1}, x_{1}\right]$ and $f\left(\left[x_{0}, x_{s}\right]\right) \supset\left[x_{t+1}, x_{1}\right]$ and $f\left(\left[x_{t+1}, x_{1}\right]\right)$ $\supset\left[x_{r}, x_{0}\right] \cup\left[x_{0}, x_{s}\right]$. This gives the existence of periodic points of all even periods.

Case 2: $n>2$. We may assume that $x_{2} \geqslant x_{0}$. If $x_{3} \leqslant x_{2}$ then there is no division, for $\left(x_{0}, x_{1}, \ldots, x_{n}\right)$ and we get a periodic point of an odd $(\neq 1)$ period. Hence we may assume $x_{2}<x_{3}$. If $d<2$ then we can use Proposition 3.5. If $p<2$ then $p=0$ and we have $x_{d} \leqslant x_{0}<x_{1}$ and there is a periodic point of period $d$. For $p, d \geqslant 2$ we have $x_{n} \leqslant x_{2}<x_{3}$. The induction hypothesis can be applied for $y_{0}=x_{2}, \ldots, y_{n-2}=$ $x_{n}$ using $n-2$ instead of $n$.

4. Examples. In this section, we shall give some examples to show that under the assumptions of our theorems we cannot obtain stronger results. In every example we shall specify only one trajectory. To be more precise, we only specify the ordering of points of this trajectory. We assume that the interval on which $f$ acts is the smallest interval containing that trajectory, and that $f$ is monotone on the components of the complement of the trajectory.

The proofs of nonexistence of certain periodic points will not be given here. The reader may check these by himself by using the method of [2], that is, by making a related graph and investigating its loops. The methods of [2] also allow us to compute the topological entropy of these examples.

EXAMPLE 1. $x_{n-1}<x_{n-3}<\cdots<x_{4}<x_{2}<x_{0}=x_{n}<x_{1}<x_{3} \cdots<x_{n-4}<$ $x_{n-2}$.

This is a classical example given by Sharkovski [5]. Here $x_{0}$ is a periodic point of period $n$, with $n \geqslant 5$ and odd, and there is no periodic point of period $n-2$. The topological entropy of this example is well known (see [2]).

EXAMPLE 2. Writing $n_{*}=n / 2, x_{n-1}<_{n_{*}-1}<x_{n-3}<x_{n_{*}-3}<\cdots<x_{n_{*}+2}<x_{2}$ $<x_{n_{*}}<x_{0}=x_{n}<x_{1}<x_{n_{*}+1}<x_{3}<x_{n_{*}+3}<\cdots<x_{n_{*}-4}<x_{n-4}<x_{n_{*}-2}<x_{n-2}$.

Here $x_{0}$ is a periodic point of period $n$, with $n$ even and $n / 2$ odd and $n \geqslant 10$. There is no division for $\left(x_{0}, x_{1}, \ldots, x_{n}\right)$. For this example, there is no periodic point 
of period $n / 2-2$. The topological entropy is the same as in Example 1 for $n_{*}$ instead of $n$.

EXAMPLE 3. Writing $n_{*}$ for $n / 2, x_{n_{*}}<x_{n-1}<x_{n_{*}-2}<x_{n-3}<\cdots<x_{4}<x_{n_{*}+3}$ $<x_{2}<x_{n_{*}+1}<x_{0}=x_{n}<x_{1}<x_{n_{*}+2}<x_{3}<\cdots<x_{n-4}<x_{n_{*}-3}<x_{n-2}<x_{n_{*}-1}$.

Here $x_{0}$ is a periodic point of period $n$, with $n \geqslant 8$ and even, and $n / 2$ is even. There is no division for $\left(x_{0}, x_{1}, \ldots, x_{n}\right)$. For this example, there is no periodic point of period $n / 2-1$. The topological entropy is $\log \lambda_{n}$ where $\lambda_{n}$ is the largest root of the polynomial $x^{n}-2 x^{n-2}-2 x^{n / 2-1}-1$.

EXAMPLE 4. $x_{2}<x_{0}<x_{3}=x_{4}<x_{1}$.

This is an example of $x_{n} \leqslant x_{0}<x_{1}$ and $x_{d} \leqslant x_{p}$ with $d$ odd and $p$ even and there is no periodic point of any odd $(\neq 1)$ period. The topological entropy is $\frac{1}{2} \log 2$.

The topological entropies in Examples 1,2 and 4 are the smallest possible for such examples. It is not known whether it is so with Example 3.

Notice that no special example is needed for Proposition 3.5. An example can be derived from Example 1 by choosing appropriate $n, d, p$.

In [4], we prove that if $x_{n} \leqslant x_{0}<x_{1}$ or $x_{n} \geqslant x_{0}>x_{1}$ for $n$ odd $(\neq 1)$, then there exists a periodic point of odd period $(\neq 1)$. One can ask if it is possible to prove that this result covers all sequences (that imply there is an odd period) in the sense that Theorem 2.4 shows Theorem 2.3 covers all sequences. The following examples shows that the answer is negative and shows there are sequences covered by Theorem 2.3 that are not covered by the results in [4].

EXAMPLE 5. $x_{6}<x_{0}<x_{4}<x_{3}=x_{7}<x_{5}<x_{2}<x_{1}$.

\section{REFERENCES}

1. L. Block, Periodic orbits of continuous mappings of the circle (preprint).

2. L. Block, J. Guckenheimer, M. Misiurewicz and L. S. Young, Periodic points and topological entropy of one dimensional maps, Global Theory of Dynamical Systems, Lecture Notes in Math., vol. 819, Springer-Verlag, Berlin and New York, 1980, pp. 18-34.

3. T. Y. Li and James A. Yorke, Period three implies chaos, Amer. Math. Monthly 82 (1975), 985-992.

4. T. Y. Li, M. Misiurewicz, G. Pianigiani and J. A. Yorke, Odd chaos (preprint).

5. A. N. Sharkovski, Coexistence of cycles of a continuous map of a line into itself, Ukrain. Mat. Ž. 16 (1964), 61-71.

Department of Mathematics, Michigan State University, East Lansing, Michigan 48824

INSTITUTE OF Mathematics, WaRSaW UNIVERSITy, 00-901 WarsaW, POLAND

Istituto Mat. “Ulisse Dini”, Viale Morgagni 67/A, I 50134 Firenze, Italy

Molecular Physics Building, University of Maryland, College Park, Maryland 20742 